\title{
Traditional Chinese Medicine Herbs and Gut Microbiota
}

\author{
Zheng-Yuan Su1 ${ }^{*}$, Zi-Han Lin ${ }^{1}$, Pei-Ju Chen ${ }^{2}$ and Yen-Chen Tung ${ }^{3}$ \\ ${ }^{1}$ Department of Bioscience Technology, Chung Yuan Christian University, Taiwan \\ ${ }^{2}$ Department of Infant and Child Educare, Cardinal Tien Junior College of Healthcare \\ and Management, Taiwan \\ ${ }^{3}$ Institute of Food Science and Technology, National Taiwan University, Taiwan
}

\section{Review Article \\ Volume 2 Issue 1}

Received Date: June 15, 2019

Published Date: June 26, 2019

DOI: $10.23880 /$ aabsc-16000133

*Corresponding author: Zheng-Yuan Su, Department of Bioscience Technology, Chung Yuan Christian University, Taoyuan City 32023, Taiwan, Tel: +886-3-2653527; Email: zysu@cycu.edu.tw

\begin{abstract}
The gut microbiota plays an important role in the human body, for example, they are responsible for the metabolism of some dietary factors. Many diseases are related to the imbalances in the gut microbiota. Accumulating evidence shows that traditional Chinese medicine herbs may prevent or treat some diseases through interacting with gut microbiota. Oral administration of Chinese herbs may change the composition of intestinal flora, and the ingredients in Chinese herbs can be catalyzed into bioactive metabolites by some intestinal microbes, resulting in their pharmacological effects. However, the interaction between Chinese herbs and gut microbiota remains unclear. One of the main challenges is the complexity and diversity of the components of Chinese herbs and intestinal flora. Recently, the correction among diseases, gut microbiota, and Chinese herbs through many methodologies are studied by using novel methods and techniques, such as high-throughput gene sequencing, real-time quantitative PCR, and metabolomics analysis as well as the multivariate data statistics. In the future, the clarified mechanism will open a new avenue for the prevention or treatment of diseases by taking Chinese herbs which regulate the composition of gut microbiota, which may achieve the goals of the personalized medicine.
\end{abstract}

Keywords: Chinese herbs; Gut microbiota; Health; Metabolism

\section{Gut Microbiota}

The intestinal system refers to the digestive tract starting from the pylorus of the stomach to the anus in the digestive system and mainly includes the small intestine, large intestine, and rectum. The small intestine is an important digestive organ in the human body and performs the functions of absorption, excretion, and immunity. It is mainly responsible for the digestion and absorption of most substances, such as sugar, lipids, protein, and vitamins. The large intestine is mainly responsible for the absorption of water in the food residue, and the food residue is excreted through the rectum. The intestines can secrete digestive juices containing nutrient-degrading enzymes and bile to assist digestion. The intestinal flora may also assist in the digestion, absorption, or metabolism of substances in certain diets [1].

At present, about $10^{14}$ bacterial species are known to be symbiotic with the human body, accounting for about 1-2 $\mathrm{Kg}$ of body weight, but the number of cells 
constituting the human body is only about $10^{13}$ [2]. The number of different genes of human intestinal bacteria is higher than 3 million, but the human genome consists of only about 22,000 genes [3]. It is known that there are at least 1000 kinds of bacteria in the intestines of healthy adults, mainly consisting of four types: Firmicutes (38.8\%), Bacteroidetes (27.8\%), Actinobacteria (8.2\%), and Proteobacteria (2.1\%); however, the proportion is different in children and the elderly, and various lifestyle also changes the composition of the intestinal flora [4]. The intestinal flora can be roughly divided into good bacteria, bad bacteria, and neutral bacteria. Many studies have found that the intestinal flora has a great influence on human health. The development of many diseases, including intestinal bacterial infections, inflammatory bowel disease, colorectal cancer, liver cancer, is often accompanied by an imbalance of intestinal bacteria [5]. Therefore, to study more about the relationship between the intestinal flora and health may assess the health status and prevent/treat diseases in humans by analyzing the profile of gut microbiota in the future [6].

\section{Gut Microbiota and Health}

In recent years, some studies have confirmed the association of certain intestinal bacteria with specific diseases. For example, the presence of Bacteroides in the intestine can drive $\mathrm{CD}^{+} \mathrm{T}$ cells to the intestinal tract to inhibit the development of inflammatory bowel disease (IBD) [7]. When Lactobacillus reuteri is implanted into mice treated with tryptophan, $L$. reuteri can metabolize trytophan into indole-3-lactic acid which promotes the transformation of intestinal epithelial $\mathrm{CD}^{+}{ }^{+} \mathrm{T}$ cells into immunoactive $\mathrm{CD} 4{ }^{+} \mathrm{CD} 8 \alpha \alpha^{+} \mathrm{T}$ cells [8]. The bioactivities of Akkermansia muciniphila are known to prevent obesity, diabetes, and non-alcoholic fatty liver (NAFLD), etc $[9,10]$. An implantation of $A$. muciniphila in the intestinal tract can improve the resistance in cancer immunotherapy in mice [11]. Bacteroides thetaiotaomicron, Faecalibacterium prausnitzii, and Holdemania filiformis can also respond to cancer immunotherapy in patients with malignant melanoma [12]. Among them, B. thetaiotaomicron can reduce the concentration of serum glutamic acid in mice and increase lipid hydrolysis and fatty acid oxidation in adipocytes, thereby reducing fat accumulation and delaying weight gain [13]. In addition, it found that a highsalt diet reduces the number of Lactobacillus murinus in mice and humans, thereby increasing the number of proinflammatory $\mathrm{T}$ helper $17\left(\mathrm{~T}_{\mathrm{H}} 17\right)$ cells; however, oral administration of L. murinus can reduce the number of TH17 cells and decrease the blood pressure in the hypertensive mice [14].
One of the hottest topics in biomedical research is to explore the effects of gut microbiota on the development of nervous system and cognitive functions [15]. Accumulating evidence suggests a crucial rol of intestinal flora in the progression of of Alzheimer's disease. As compared with the normal human intestinal flora, there are fewer Firmicutes and Bifidobacterium but more Bacteroidetes in the intestines of patients suffering from Alzheimer's disease [16]. It was also found that extracellular amyloids (known as curli fibers) secreted by Escherichia coli (strain K12) contain the main structure of curli subunit gA (CsgA) which assist in the adsorption of bacteria and the production of biofilms, which are related to pathogenicity [17]. A CsgA amyloid precursor of approximately $18 \mathrm{kDa}$ contains a pathogen-associated molecular pattern (PAMP) similar to the amyloid-beta 42 (A 342$)$ peptide and is recognized by the human immune system toll-like receptor 2 [18]. Other intestinal bacterial species, such as Bacillus, Pseudomonas, Staphylococcus, and Streptomyces may also possess bacterial amyloids [19]. Furthermore, when some genes in the gut bacteria are eliminated or mutated, it prolongs the lifespan of Caenorhabditis elegans, delays tumor progression, and reduces amyloid-beta accumulation [20].

\section{Gut Microbiota and Metabolism}

Since the intestinal flora of the human body has some genes that are responsible for the production of enzymes, they may involve in biochemical metabolism in the intestine, such as sugars, proteins, fatty acids, cholesterol, vitamins, and other foreign compounds, and then the body may further absorbs theese metabolites [21]. For example, in addition to dietary fiber, some flora can metabolize short-chain fatty acids into n-butyrate, acetate, butyrate, isobutyrate, propionate, valerate, isovalerate, hexanoate, etc $[21,22]$. These flora may be the clormicials IV and XIVa of the Firmicutes, including Eubacterium, Roseburia, Faecalibacterium, and Coprococcus, which may cause decreased intestinal $\mathrm{pH}$, inhibit growth of pathogens, provide energy to intestinal cells; improve obesity, insulin resistance, type 2 diabetes; and prevent colorectal cancer [23]. Other gut bacteria can metabolize amino acids into bioactive products. For example, $L$ tryptophan can be metabolized into a neurotransmitter tryptamine or an antioxidant indole3-propionic [24]. Lactobacilli, Bifidobacteria, Enterobacter, Clostridium, Bacteroides and other intestinal flora can metabolize bile acid into cholate, hyocholate, deoxycholate, chenodeoxycholate, $\alpha$-muricholate, etc., which promote the absorption of lipid and ipid-soluble vitamins, maintain function of the intestinal barrier, and regulate the triglyceride, cholesterol, glucose levels and other 
functions of the body [25]. F. prausnitzii and Bifidobacterium have been shown to be involved in the metabolism of choline, thus producing methylamine, dimethylamine, trimethylamine, trimethylamine- $N$-oxide, dimethylglycine, betaine, etc., thereby regulating fat metabolism and blood sugar balance and preventiing nonalcoholic fatty liver, obesity, diabetes, and cardiovascular disease [26]. In addition, Bifidobacterium can synthesize a variety of vitamins in the intestine, including vitamin $K$, vitamin B12, folic acid, thiamine, riboflavin, etc., which provide endogenous vitamins to the body [27].

Since intestinal flora varies in different people, there may be differences in the metabolism and absorption of some food components between individuals. For example, Eubacterium coprostanoligenes in the gut microbiota in some population that can decompose $50 \%$ of dietary cholesterol in the intestines into coprostanol that is not easily absorbed by the body [28]. Heterocyclic amines, such as 2-amino3-methylimidazo [4,5-f] quinolone (IQ), are carcinogens that are easily produced during the process of baking foods, but an E. coli strain with the $\beta$ glucuronidase (uidA) gene in the intestine can transform IQ into hydrolysable IQ-glucuronide; however, some bacteria may metabolize IQ into more toxic 7-hydroxy IQ which cannot be metabolized by the E. coli strain [29]. In addition, diet and medication can also change the intestinal flora, which affects the metabolism of substances in the intestinal tract. It reported that intermittent fasting causes the changes in the composition of the intestinal flora in normal mice, which increases the levels of acetic acid and lactic acid, as well as the conversion of white fat into beige fat, thereby resulting in weight loss and liver protection; however, this phenomenon is not observed in mice with intestinal flora deficiency [30]. Another study found that a high-fat and low-carbohydrate ketogenic diet has an anti-epileptic effect, because this diet can increase the number of the intestinal bacteria, Akkermansia and Parabacteroides, thus improving the content of gamma-aminobutyric acid (GABA) which inhibits nerve conduction in the hippocampus [31]. Taken together, these studies indicate that diet does change the composition of the intestinal flora and affect the physiological condition and metabolism in mammals.

\section{Influence of Chinese Herbal Medicine on Gut Microbiota-Related Diseases}

Many studies have demonstrated that Chinese herbal medicines can alleviate many diseases, including obesity, diabetes, nonalcoholic steatohepatitis, ulcerative colitis, cancer, chronic kidney disease, Alzheimer's disease, etc.
Changing the composition of the intestinal flora could be one of the main mechanisms by which Chinese herbal medicine improves symptoms of such diseases. If Chinese herbs could promote the growth of beneficial bacteria as well as inhibit the growth of harmful bacteria/pathogenic bacteria, the host could keep healthy. The active ingredients in the Chinese herbs that promotes the growth of the intestinal flora may be carbohydrates or sugar-containing compounds, such as inulin, fructooligosaccharides, galacto-oligosaccharides, etc [32]. Intestinal bacteria can metabolize some sugars into shortchain fatty acids, which may eventually inhibit the growth of harmful bacteria /pathogenic bacteria [22-24]. Additionally, other components in Chinese herbs may also contribute to the influence on the growth of beneficial and harmful/pathogenic bacteria.

For example, oral administered with Ginseng Radix Rubra (hong shen) increases the level of lactobacillus in the intestines of rats, and Bawei Xileisan containing watermelon (Citrullus lanatus, xī guā) and Isatis indigotica (băn lán gēn) leaves and stems increases the levels of Bacteroides and Lactobacillus species in the intestines of mice, hence such Chinese herbs may prevent ulcerative colitis $[33,34]$. Some Chinese herbs have also been found to effectively inhibit harmful bacteria/pathogenic bacteria in the intestines, such as E. coli, Helicobacter pylori, Campylobacter jejuni, Enterococcus faecalis, Salmonella typhimurium, Bacillus subtilis, Klebsiella pneumoniae, Shigella dysenteriae, Pseudomonas aeruginosa, etc [35]. A Chinese herbal formula mainly composed of Artemisiae Scopariae Herba (yīn chén hāo), Polygoni Cuspidati Rhizoma (hǔ zhàng), Curcumae Longae Rhizoma (jiāng huáng), Hyperici Japonici Herba (dì ěr căo), and Gardeniae Fructus (zhī zì) can reduce the amount of bac Escherichia, Shigella, and Sporacetigenium in the intestines and improve the symptoms of nonalcoholic fatty liver [35]. In addition, mice administered Ganoderma (líng zhī) fruiting bodies were found to have increased intestinal flora comprising Parabacteroides goldsteinii, Bacteroides, Anaerotruncus colihominis, Roseburia hominis, Clostridium methylpentosum, Clostridium XIVa, Clostridium XVIII, and E. coprostanoligenes, but have a reduced flora comprising Firmicutes/Bacteriodetes, Proteobacteria, Mucispirillum schaedleri, Escherichia fergusonii, Enteroroccus, Lactococcus lactis, Clostridium lactatifermentans, and Oscillibacter valericigenes, thereby leading to a reduction of weight and fat accumulation [36]. Coptidis Rhizoma (huáng lián) extract and its active ingredient, berberine, can significantly reduce body fat, blood sugar, blood lipids, etc., in high-fat diet mice; reduce the numbers of Firmicutes and Bacteroidetes in feces; and inhibit the growth of lactobacillus in in vivo culture experiments [37]. 
Increased numbers of Bacteriodetes, Firmicutes, Bifidobacterium, Lactobacillus, Akkermansia and other bacteria were found in rats fed with Lonicerae Flos (jīn yín huā) [38]. Besides, a report indicated that the saponins in Gynostemma pentaphyllum (jiăogǔlán) could reduce colorectal carcinogenesis in $\mathrm{Apc}^{\mathrm{Min} /+}$ mice with the alternation of the intestinal microflora, including the increased numbers of Actinobacteria, Proteobacteria, Acinetobacter lwoffii, Allobaculum splD4, Bacteroides acidifaciens, Bifidobacterium pseudocatenulatum, Clostridium cocleatum, Parabacteroides distasonis, Streptococcus thermophiles, and Bacteroidetes/Firmicutes; and the reduced numbers of Firmicutes, Tenericutes, Verrucomicrobia, Cyanobacteria, Bacteroides uniformis, Deltatopotebacteria [39]. G. pentaphyllum (jiăogǔlán) saponins also inhibited tumor growth in BALB/c mice while Clostridia is decreased, and Bataproteobacteria, Erysipelotrichi, Alcaligenaceae, Erysipelotrichaceae, Clostridium, C. cocleatum, B. acidifaciens are increased in the intestine [40]. A study indicated that a Chinese herbal formula containing Rehmanniae Radix Praeparata (shú dì huáng), Corni Fructus (shān zhū yú), Moutan Cortex (mǔ dān pí), Alismatis Rhizoma (zé xiè), Dioscoreae Rhizoma (shān yào), and Poria (fú líng) improved cognitive impairment in SAMP8 mice, and increased intestinal Adlercreutzia, Anaerrotruncus, Ruminococcus, Coprococcus, etc.; but reduced Prevotella, Streptococcus, Veillonella, Bilophila, etc., which may improve Alzheimer's disease [41].

The occurrence of diseases may be related to the intestinal bacteria, and Chinese herbs can alternate the composition of intestinal flora, which contribute to the prevention and/or treatment of diseases. Therefore, it is worthy to clarify the association between the Chinese herbs and intestinal flora, and further to establish a database of such relationship. Better understaing of the interactions among the Chinese herbs, intestinal flora, and diseases will support novel approaches for the the development of health foods and drugs in the future.

\section{Gut Microbiota Metabolize the Components in Chinese Herbs}

When the Chinese herbs are administered clinically, they were often extracted with water or ethanol to form a decoction, or further concentrated into a paste, powder, or tablet. In general, most of the water extracts of Chinese herbs are hydrophilic compounds, while those with larger proportions in ethanol extracts are lipophilic compounds. Some compounds have low bioavailability and have to undergo structural transformation to form bioactive metabolites. The liver is an organ that mainly metabolizes dietary components. However, many studies have proposed that precursors of active ingredients in Chinese herbs, after coming in contact with the intestinal flora, are further metabolized by the enzymes of the flora into substances that are more easily absorbed by the intestines; hence, the biological activity of the components of the Chinese herbs is further improved [37]. There are many types of intestinal bacteria, and different kinds of bacteria in the gastrointestinal tract secrete different metabolic enzymes that may participate in the metabolism of various types of active compounds [35].

Glycoside compounds are one of the main components of Chinese herbs, and some intestinal flora can hydrolyze glycosidic bonds to form hydrophobic aglycons [42]. Intestinal flora that have been shown to be capable of hydrolyzing glycosides, including Bifidobacterium sp., Eubacterium sp., Bacteroides sp., Fusobacterium K-60, Prevotella oris, Peptostreptococcus intermedius, Clostridium perfringens, Ruminococcus sp., Clostridium innocum, Eubacterium sp., Eubacterium sp., Bifidobacterium $s p$., these can metabolize glycosylcontaining triterpenoids [43-46]. It was also found that Bacteroides distasonis, Butyrivibrio fibrisolvens, Butyrivibrio sp., Selenomonas ruminantium, Butyrivibrio sp., Bifidobacterium dentium, Fusobacterium, Escherichia, Enterococcus sp., Clostridium coccoides, and Bifidobacterium infantis can hydrolyze glycosidic linkages of glycosyl-containing phenolic compounds [47-50]. In addition, some compounds are further broken down into smaller compounds by intestinal flora. For example, rutin can be metabolized into protocatechuic acid, 3hydroxyphenyl-acetic acid, 3-(3-hydroxyphenyl)propionic acid, 3,4-dihydroxyphenyl-acetic acid, 3hydroxyhippuric acid, etc., which are small compounds that are absorbed by the large intestine [51].

Some Chinese herbs contain a large number of alkaloids. For example, aconite, a Chinese Aconiti Radix Wutou (wū tóu), can be converted into lipoaconitine by Bacteroides fragilis, K. pneumoniae, and Clostridium butyricum in the human intestinal tract [52]. The berberine component of C. Rhizoma (huáng lián) can be metabolized into dihydroberberine in the intestine of rats [53]. The bacteria that catalyze the transformation reaction of berberine include Staphylococcus aureus, Enterococcus faecium, E. faecalis, Enterobacter cloacae, E. coli, Staphylococcus, P. aeruginosa, K. pneumonia, Proteus mirabilis, Acinetobacter baumannii, Lactobacillus casei, Lactobacillus acidophilus, Bifidobacterium longum, Bifidobacterium breve, etc. [53]. In addition, special bacteria capable of metabolizing rhein, a steroid 
compound in Rhei Radix et Rhizoma (dà huáng), into rheinanthrone were found in the intestinal tract, namely Bacteroides sp. RHEIN-I and Bacteroides sp. RHEIN-II [54]. Blautia sp. AUH-JLD56 in the human small intestine converts arctiin, a lignin in Arctii Fructus (niú bàng), into arctigenin and (-)-3'-desmethylarctigenin [55]. Ellagitannins in many Chinese herbs can be broken down into small molecules urolithins by the human intestinal Gordonibacter urolithinfaciens sp. nov. and Gordonibacter pamelaeae DSM 19378T [56]. It has also been found that Eubacterium sp. strain SDG-2, Slackia equolifaciens JCM 16059, Slackia isoflavoniconvertens JCM 16137, Adlercreutzia equolifaciens JCM 14793, Asaccharobacter celatus JCM 14811 can metabolize condensed tannins in Chinese herbs into several derivatives, such as valerolactone, benzoic acid, phenylacetic acid, phenylpropionic acid, and phenyllactic acid [57].

\section{The Prospect of Gut Microbiota and Chinese Herbs}

In the recent years, many researches on the interaction between Chinese herbs and intestinal flora as well as the health of organisms have attracted the attention of many scholars in order to understand how Chinese herbs can prevent or treat diseases [5,6]. One of the directions of research related to Chinese herbs to prevent/treat diseases by influencing intestinal bacteria is to explore the mechanisms by which Chinese herbs could change the intestinal flora, such as promoting the growth of probiotics-producing bacteria or inhibiting the growth of harmful bacteria/pathogenic bacteria. A good balance of the intestinal flora promotes the health of the host; hence, it is necessary to analyze the Chinese herbal ingredients that may affect the growth of the intestinal flora $[32,41]$. The intestinal flora may also affect the absorption, metabolism, and transformation of Chinese medicines administered orally and changes the efficacy of traditional Chinese medicine.

However, due to the complexity and diversity of the components of traditional Chinese medicine herbs and their endogenous metabolites, studies on the structure of the intestinal flora and the co-metabolism of the host have not been reported and need to be resolved. Previous studies on the intestinal flora and host metabolism have mostly been conducted as separate studies, that is, the changes of intestinal flora and the effects of bacterial changes on host metabolism have been studied separately. These studies do not clearly explain the impact of a specific flora change on host metabolism. In addition, since the intestinal flora metabolizes the components of Chinese herbs into biologically active products $[42,52,53]$. In order to understand the interaction between intestinal bacteria and traditional Chinese medicine, studying only a single chemical component cannot meet research needs. Therefore, it is necessary to identify all chemical substances involved in the metabolic process, directly measure the flora in the intestinal tract, and provide accurate research models.

Recently, the microbiome has been extensively studied using high-throughput gene sequencing, real-time quantitative PCR, and metabolomics methods and techniques as well as the multivariate data statistics. Among the many variables, the main variables, which are significant and have high modeling abilities, are selected to construct a biological effect model with good stability and generalization ability. The specific biological effects of Chinese medicine, composition of the intestinal flora, and their interaction on host health can thus be analyzed. However, further studies are necessary to find biomarkers and endogenous metabolites related to specific biological effects and to explore the biological mechanism and material basis of traditional Chinese medicine through the study of co-metabolism between intestinal flora and host. Studies on the mutually beneficial symbiotic relationship and the role of intestinal flora on the health of the host and its role in the development of the disease will have a very important impact on human health.

\section{Conflict of Interest}

Declarations of interest: none.

\section{Acknowledgments}

This work is supported in part by institutional funds and by MOST 106-2311-B-033-002-MY3 from Ministry of Science and Technology.

\section{References}

1. Cani PD, Delzenne NM (2007) Gut microflora as a target for energy and metabolic homeostasis. Curr Opin Clin Nutr Metab Care 10(6): 729-734.

2. Huttenhower C, Gevers D, Knight R, Abubucker S, Badger JH, et al. (2012) Structure, function and diversity of the healthy human microbiome. Nature 486(7402): 207-214.

3. Ursell LK, Metcalf JL, Parfrey LW, Knight R (2012) Defining the human microbiome. Nutr Rev 70: S38S44. 
4. D'Argenio V, Salvatore F (2015) The role of the gut microbiome in the healthy adult status. Clin Chim Acta 451: 97-102.

5. Tojo R, Suarez A, Clemente MG, de los Reyes-Gavilan CG, Margolles A, et al. (2014) Intestinal microbiota in health and disease: Role of bifidobacteria in gut homeostasis. World J Gastroenterol 20(41): 1516315176.

6. Vandeputte D, Kathagen G, D'Hoe K, Vieira-Silva S, Valles-Colomer M, et al. (2017) Quantitative microbiome profiling links gut community variation to microbial load. Nature 551(7681): 507-511.

7. Nanjundappa RH, Ronchi F, Wang JG, ClementeCasares X, Yamanouchi J, et al. (2017) A Gut Microbial Mimic that Hijacks Diabetogenic Autoreactivity to Suppress Colitis. Cell 171(3): 655-667.

8. Cervantes-Barragan L, Chai JN, Tianero MD, Di Luccia B, Ahern PP, et al. (2017) Lactobacillus reuteri induces gut intraepithelial CD4(+)CD8 alpha alpha(+) T cells. Science 357(6353): 806-810.

9. Moreira GV, Azevedo FF, Ribeiro LM, Santos A, Guadagnini D, et al. (2018) Liraglutide modulates gut microbiota and reduces NAFLD in obese mice. J Nutr Biochem 62: 143-154.

10. Zhu A, Chen J, Wu P, Luo M, Zeng Y, et al. (2017) Cationic Polystyrene Resolves Nonalcoholic Steatohepatitis, Obesity, and Metabolic Disorders by Promoting Eubiosis of Gut Microbiota and Decreasing Endotoxemia. Diabetes 66(8): 2137-2143.

11. Routy B, Le Chatelier E, Derosa L, Duong CPM, Alou MT, et al. (2018) Gut microbiome influences efficacy of PD-1-based immunotherapy against epithelial tumors. Science 359(6371): 91-97.

12. Frankel AE, Coughlin LA, Kim J, Froehlich TW, Xie Y, et al. (2017) Metagenomic Shotgun Sequencing and Unbiased Metabolomic Profiling Identify Specific Human Gut Microbiota and Metabolites Associated with Immune Checkpoint Therapy Efficacy in Melanoma Patients. Neoplasia 19(10): 848-855.

13. Liu RX, Hong J, Xu XQ, Feng Q, Zhang DY, et al. (2017) Gut microbiome and serum metabolome alterations in obesity and after weight-loss intervention. Nat Med 23(7): 859-868.
14. Wilck N, Matus MG, Kearney SM, Olesen SW, Forslund $\mathrm{K}$, et al. (2017) Salt-responsive gut commensal modulates TH17 axis and disease. Nature 551(7682): 585-589.

15. Pistollato F, Cano SS, Elio I, Vergara MM, Giampieri F, et al. (2016) Role of gut microbiota and nutrients in amyloid formation and pathogenesis of Alzheimer disease. Nutr Rev 74(10): 624-634.

16. Vogt NM, Kerby RL, Dill-McFarland KA, Harding SJ, Merluzzi AP, et al. (2017) Gut microbiome alterations in Alzheimer's disease. Sci Rep 7(1): 13537.

17. Schwartz K, Boles BR (2013) Microbial amyloidsfunctions and interactions within the host. Curr Opin Microbiol 16(1): 93-99.

18. Zhou ZD, Chan $\mathrm{CH}, \mathrm{Ma} \mathrm{QH}, \mathrm{Xu} \mathrm{XH}$, Xiao ZC, et al. (2011) The roles of amyloid precursor protein (APP) in neurogenesis: Implications to pathogenesis and therapy of Alzheimer disease. Cell Adh Migr 5(4): 280-292.

19. Han B, Sivaramakrishnan P, Lin CCJ, Neve IAA, He JQ, et al. (2017) Microbial Genetic Composition Tunes Host Longevity. Cell 169(7): 1249-1262.

20. Nicholson JK, Holmes E, Kinross J, Burcelin R, Gibson G, et al. (2012) Host-Gut Microbiota Metabolic Interactions. Science 336(6086): 1262-1267.

21. Wong JMW, de Souza R, Kendall CWC, Emam A, Jenkins DJA (2006) Colonic health: Fermentation and short chain fatty acids. J Clin Gastroenterol 40(3): 235-243.

22. Louis P, Young P, Holtrop G, Flint HJ (2010) Diversity of human colonic butyrate-producing bacteria revealed by analysis of the butyryl-CoA: acetate CoAtransferase gene. Environ Microbiol 12(2): 304-314.

23. Cani PD (2017) Gut cell metabolism shapes the microbiome. Science 357(6351): 548-549.

24. Wikoff WR, Anfora AT, Liu J, Schultz PG, Lesley SA, et al. (2009) Metabolomics analysis reveals large effects of gut microflora on mammalian blood metabolites. $\mathrm{P}$ Natl Acad Sci USA 106(10): 3698-3703.

25. Staley C, Weingarden AR, Khoruts A, Sadowsky MJ (2017) Interaction of gut microbiota with bile acid metabolism and its influence on disease states. Appl Microbiol Biotechnol 101(1): 47-64. 
26. Singh RK, Chang HW, Yan D, Lee KM, Ucmak D, et al. (2017) Influence of diet on the gut microbiome and implications for human health. J Transl Med 15(1): 73.

27. Thakur K, Tomar SK, De S (2016) Lactic acid bacteria as a cell factory for riboflavin production. Microb Biotechnol 9(4): 441-451.

28. Koppel N, Rekdal VM, Balskus EP (2017) Chemical transformation of xenobiotics by the human gut microbiota. Science 356(6344).

29. Humblot C, Murkovic M, Rigottier-Gois L, Bensaada M, Bouclet A, et al. (2007) beta-Glucuronidase in human intestinal microbiota is necessary for the colonic genotoxicity of the food-borne carcinogen 2-amino-3methylimidazo[4,5-f]quinoline in rats. Carcinogenesis 28(11): 2419-2425.

30. Li GL, Xie C, Lu SY, Nichols RG, Tian Y, et al. (2017) Intermittent Fasting Promotes White Adipose Browning and Decreases Obesity by Shaping the Gut Microbiota. Cell Metab 26(4): 672-685.

31. Olson CA, Vuong HE, Yano JM, Liang QXY, Nusbaum DJ, et al. (2018) The Gut Microbiota Mediates the Anti-Seizure Effects of the Ketogenic Diet. Cell 173(7): 1728-1741.

32. Roberfroid M, Gibson GR, Hoyles L, McCartney AL, Rastall R, et al. (2010) Prebiotic effects: metabolic and health benefits. Br J Nutr 104: S1-S63.

33. Guo MZ, Ding S, Zhao CH, Gu XX, He XY, et al. (2015) Red Ginseng and Semen Coicis can improve the structure of gut microbiota and relieve the symptoms of ulcerative colitis. J Ethnopharmacol 162: 7-13.

34. Wen J, Teng BX, Yang PR, Chen XJ, Li CH, et al. (2016) The potential mechanism of Bawei Xileisan in the treatment of dextran sulfate sodium-induced ulcerative colitis in mice. J Ethnopharmacol 188: 31 38.

35. Xu J, Chen HB, Li SL (2017) Understanding the Molecular Mechanisms of the Interplay Between Herbal Medicines and Gut Microbiota. Med Res Rev 37(5): 1140-1185.

36. Chang CJ, Lin CS, Lu CC, Martel J, Ko YF, et al. (2015) Ganoderma lucidum reduces obesity in mice by modulating the composition of the gut microbiota. Nature Communications. 6.
37. Xie WD, Gu DY, Li JN, Cui K, Zhang YO (2011) Effects and Action Mechanisms of Berberine and Rhizoma coptidis on Gut Microbes and Obesity in High-Fat Diet-Fed C57BL/6J Mice. Plos One 6(9): e24520.

38. Xu J, Lian FM, Zhao LH, Zhao YF, Chen XY, et al. (2015) Structural modulation of gut microbiota during alleviation of type 2 diabetes with a Chinese herbal formula. Isme J 9(3): 552-562.

39. Chen L, Brar MS, Leung FCC, Hsiao WLW (2016) Triterpenoid herbal saponins enhance beneficial bacteria, decrease sulfate-reducing bacteria, modulate inflammatory intestinal microenvironment and exert cancer preventive effects in Apc(Min/+) mice. Oncotarget 7(21): 31226-31242.

40. Chen L, Tai WCS, Brar MS, Leung FCC, Hsiao WLW (2015) Tumor Grafting Induces Changes of Gut Microbiota in Athymic Nude Mice in the Presence and Absence of Medicinal Gynostemma Saponins. Plos One 10(5): e0126807.

41. Wang JH, Ye FQ, Cheng XR, Zhang XR, Liu F, et al. (2016) The Effects of LW-AFC on Intestinal Microbiome in Senescence-Accelerated Mouse Prone 8 Strain, a Mouse Model of Alzheimer's Disease. Journal of Alzheimers Disease 53(3): 907-919.

42. Steer TE, Johnson IT, Gee JM, Gibson GR (2003) Metabolism of the soyabean isoflavone glycoside genistin in vitro by human gut bacteria and the effect of prebiotics. Br J Nutr 90(3): 635-642.

43. Xiang C, Qiao X, Wang Q, Li R, Miao W, et al. (2011) From single compounds to herbal extract: a strategy to systematically characterize the metabolites of licorice in rats. Drug Metab Dispos 39(9): 1597-1608.

44. Wan JY, Liu P, Wang HY, Qi LW, Wang CZ, et al. (2013) Biotransformation and metabolic profile of American ginseng saponins with human intestinal microflora by liquid chromatography quadrupole time-of-flight mass spectrometry. J Chromatogr A 1286: 83-92.

45. Wang HY, Hua HY, Liu XY, Liu JH, Yu BY (2014) In vitro biotransformation of red ginseng extract by human intestinal microflora: metabolites identification and metabolic profile elucidation using LC-Q-TOF/MS. J Pharm Biomed Anal 98: 296-306.

46. Wang G, Fu H, Ye W, Zheng X, Xiao J, Kang D, et al. (2016) Comprehensive characterization of the in vitro and in vivo metabolites of ziyuglycoside I in rat 
microsome, intestinal flora, excretion specimen and fresh tissues based on LC-Q-TOF/MS. J Pharm Biomed Anal 128: 191-200.

47. Bang SH, Hyun YJ, Shim J, Hong SW, Kim DH (2015) Metabolism of Rutin and Poncirin by Human Intestinal Microbiota and Cloning of Their Metabolizing alpha-L-Rhamnosidase from Bifidobacterium dentium. J Microbiol Biotechnol 25(1): 18-25.

48. Yang J, Qian DW, Guo JM, Jiang S, Shang EX, et al. (2013) Identification of the major metabolites of hyperoside produced by the human intestinal bacteria using the ultra performance liquid chromatography/quadrupole-time-of-flight mass spectrometry. J Ethnopharmacol 147(1): 174-179.

49. Lu XY, Sun DL, Chen ZJ, Chen T, Li LP, et al. (2010)Relative contribution of small and large intestine to deglycosylation and absorption of flavonoids from Chrysanthemun morifolium extract. J Agric Food Chem 58(19): 10661-10667.

50. Marin L, Miguelez EM, Villar CJ, Lombo F (2015) Bioavailability of Dietary Polyphenols and Gut Microbiota Metabolism: Antimicrobial Properties. Biomed Research International 2015: 905215.

51. Rechner AR, Smith MA, Kuhnle G, Gibson GR, Debnam ES, et al. (2004) Colonic metabolism of dietary polyphenols: Influence of structure on microbial fermentation products. Free Radical Biol Med 36(2): 212-225.
52. Kawata Y, Cho-mei MA, MM R, Nakamura N, Wang H, et al. (1999) Conversion of aconitine to lipoaconitine by human intestinal bacteria and their antinociceptive effects in mice. J Trad Med 16(1): 1523.

53. Feng R, Shou JW, Zhao ZX, He CY, Ma C, et al. (2015) Transforming berberine into its intestine-absorbable form by the gut microbiota. Sci Rep 5 .

54. Ragab MM, Nishimoto E, Akao T, Hattori M (2001) Human intestinal Bacteroides spp. RHEIN-I and RHEIN-II capable of transforming rhein to rheinanthrone, induce rhein-dependent diarrhea in rats. J Trad Med 18(4): 169-176.

55. Liu MY, Li M, Wang XL, Liu P, Hao QH, et al. (2013) Study on Human Intestinal Bacterium Blautia sp AUHJLD56 for the Conversion of Arctigenin to (-)-3 'Desmethylarctigenin. J Agr Food Chem 61(49): 12060-12065.

56. Selma MV, Beltran D, Garcia-Villalba R, Espin JC, Tomas-Barberan FA. (2014) Description of urolithin production capacity from ellagic acid of two human intestinal Gordonibacter species. Food Funct 5(8): 1779-1784.

57. Takagaki A, Nanjo F (2015) Biotransformation of (-)Epigallocatechin and (-)-Gallocatechin by Intestinal Bacteria Involved in Isoflavone Metabolism. Biol Pharm Bull 38(2): 325-330. 